\title{
FOIL it! Find One mismatch between Image and Language caption
}

\author{
Ravi Shekhar, Sandro Pezzelle, Yauhen Klimovich, \\ Aurélie Herbelot, Moin Nabi, Enver Sangineto, Raffaella Bernardi \\ University of Trento \\ \{firstname.lastname\}@unitn.it
}

\begin{abstract}
In this paper, we aim to understand whether current language and vision (LaVi) models truly grasp the interaction between the two modalities. To this end, we propose an extension of the MSCOCO dataset, FOIL-COCO, which associates images with both correct and 'foil' captions, that is, descriptions of the image that are highly similar to the original ones, but contain one single mistake ('foil word'). We show that current LaVi models fall into the traps of this data and perform badly on three tasks: a) caption classification (correct vs. foil); b) foil word detection; c) foil word correction. Humans, in contrast, have near-perfect performance on those tasks. We demonstrate that merely utilising language cues is not enough to model FOIL-COCO and that it challenges the state-of-the-art by requiring a fine-grained understanding of the relation between text and image.
\end{abstract}

\section{Introduction}

Most human language understanding is grounded in perception. There is thus growing interest in combining information from language and vision in the NLP and AI communities.

So far, the primary testbeds of Language and Vision (LaVi) models have been 'Visual Question Answering' (VQA) (e.g. Antol et al. (2015); Malinowski and Fritz (2014); Malinowski et al. (2015); Gao et al. (2015); Ren et al. (2015)) and 'Image Captioning' (IC) (e.g. Hodosh et al. (2013); Fang et al. (2015); Chen and Lawrence Zitnick (2015); Donahue et al. (2015); Karpathy and Fei-Fei (2015); Vinyals et al. (2015)). Whilst some models have seemed extremely successful on those tasks, it remains unclear how the reported results should be interpreted and what those

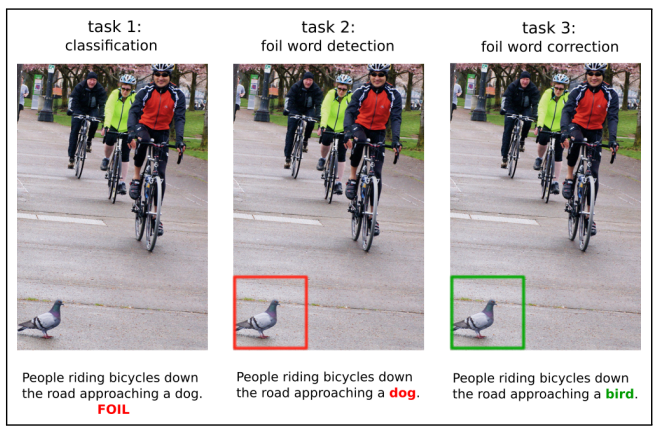

Figure 1: Is the caption correct or foil (T1)? If it is foil, where is the mistake (T2) and which is the word to correct the foil one (T3)?

models are actually learning. There is an emerging feeling in the community that the VQA task should be revisited, especially as many current dataset can be handled by 'blind' models which use language input only, or by simple concatenation of language and vision features (Agrawal et al., 2016; Jabri et al., 2016; Zhang et al., 2016; Goyal et al., 2016a). In IC too, Hodosh and Hockenmaier (2016) showed that, contrarily to what prior research had suggested, the task is far from been solved, since IC models are not able to distinguish between a correct and incorrect caption.

Such results indicate that in current datasets, language provides priors that make LaVi models successful without truly understanding and integrating language and vision. But problems do not stop at biases. Johnson et al. (2016) also point out that current data 'conflate multiple sources of error, making it hard to pinpoint model weaknesses', thus highlighting the need for diagnostic datasets. Thirdly, existing IC evaluation metrics are sensitive to n-gram overlap and there is a need for measures that better simulate human judgments (Hodosh et al., 2013; Elliott and Keller, 2014; Anderson et al., 2016).

Our paper tackles the identified issues by proposing an automatic method for creating a 
large dataset of real images with minimal language bias and some diagnostic abilities. Our dataset, FOIL (Find One mismatch between Image and Language caption), ${ }_{1}^{1}$ consists of images associated with incorrect captions. The captions are produced by introducing one single error (or 'foil') per caption in existing, human-annotated data (Figure 1). This process results in a challenging error-detection/correction setting (because the caption is 'nearly' correct). It also provides us with a ground truth (we know where the error is) that can be used to objectively measure the performance of current models.

We propose three tasks based on widely accepted evaluation measures: we test the ability of the system to a) compute whether a caption is compatible with the image (T1); b) when it is incompatible, highlight the mismatch in the caption (T2); c) correct the mistake by replacing the foil word (T3).

The dataset presented in this paper (Section 3) is built on top of MS-COCO (Lin et al., 2014), and contains 297,268 datapoints and 97,847 images. We will refer to it as FOIL-COCO. We evaluate two state-of-the-art VQA models: the popular one by Antol et al. (2015), and the attention-based model by Lu et al. (2016), and one popular IC model by (Wang et al., 2016). We show that those models perform close to chance level, while humans can perform the tasks accurately (Section 4). Section 5 provides an analysis of our results, allowing us to diagnose three failures of LaVi models. First, their coarse representations of language and visual input do not encode suitably structured information to spot mismatches between an utterance and the corresponding scene (tested by T1). Second, their language representation is not finegrained enough to identify the part of an utterance that causes a mismatch with the image as it is (T2). Third, their visual representation is also too poor to spot and name the visual area that corresponds to a captioning error (T3).

\section{Related Work}

The image captioning (IC) and visual question answering (VQA) tasks are the most relevant to our work. In IC (Fang et al., 2015; Chen and Lawrence Zitnick, 2015; Donahue et al., 2015; Karpathy and Fei-Fei, 2015; Vinyals et al., 2015;

\footnotetext{
${ }^{1}$ The dataset is available from https:// foilunitn. github.io/
}

Wang et al., 2016), the goal is to generate a caption for a given image, such that it is both semantically and syntactically correct, and properly describes the content of that image. In VQA (Antol et al., 2015; Malinowski and Fritz, 2014; Malinowski et al., 2015; Gao et al., 2015; Ren et al., 2015), the system attempts to answer open-ended questions related to the content of the image. There is a wealth of literature on both tasks, but we only discuss here the ones most related to our work and refer the reader to the recent surveys by (Bernardi et al., 2016; Wu et al., 2016).

Despite their success, it remains unclear whether state-of-the-art LaVi models capture vision and language in a truly integrative fashion. We could identify three types of arguments surrounding the high performance of LaVi models:

(i) Triviality of the LaVi tasks: Recent work has shown that LaVi models heavily rely on language priors (Ren et al., 2015; Agrawal et al., 2016; Kafle and Kanan, 2016). Even simple correlation and memorisation can result in good performance, without the underlying models truly understanding visual content (Zhou et al., 2015; Jabri et al., 2016; Hodosh and Hockenmaier, 2016). Zhang et al. (2016) first unveiled that there exists a huge bias in the popular VQA dataset by Antol et al. (2015): they showed that almost half of all the questions in this dataset could be answered correctly by using the question alone and ignoring the image completely. In the same vein, Zhou et al. (2015) proposed a simple baseline for the task of VQA. This baseline simply concatenates the Bag of Words (BoW) features from the question and Convolutional Neural Networks (CNN) features from the image to predict the answer. They showed that such a simple method can achieve comparable performance to complex and deep architectures. Jabri et al. (2016) proposed a similar model for the task of multiple choice VQA, and suggested a cross-dataset generalization scheme as an evaluation criterion for VQA systems. We complement this research by introducing three new tasks with different levels of difficulty, on which LaVi models can be evaluated sequentially.

(ii) Need for diagnostics: To overcome the bias uncovered in previous datasets, several research groups have started proposing tasks which involve distinguishing distractors from a groundtruth caption for an image. Zhang et al. (2016) introduced a binary VQA task along with a dataset 
composed of sets of similar artificial images, allowing for more precise diagnostics of a system's errors. Goyal et al. (2016a) balanced the dataset of Antol et al. (2015), collecting a new set of complementary natural images which are similar to existing items in the original dataset, but result in different answers to a common question. Hodosh and Hockenmaier (2016) also proposed to evaluate a number of state-of-the-art LaVi algorithms in the presence of distractors. Their evaluation was however limited to a small dataset (namely, Flickr30K (Young et al., 2014)) and the caption generation was based on a hand-crafted scheme using only inter-dataset distractors.

Most related to our paper is the work by Ding et al. (2016). Like us, they propose to extend the MS-COCO dataset by generating decoys from human-created image captions. They also suggest an evaluation apparently similar to our T1, requiring the LaVi system to detect the true target caption amongst the decoys. Our efforts, however, differ in some substantial ways. First, their technique to create incorrect captions (using BLEU to set an upper similarity threshold) is so that many of those captions will differ from the gold description in more than one respect. For instance, the caption two elephants standing next to each other in a grass field is associated with the decoy a herd of giraffes standing next to each other in a dirt field (errors: herd, giraffe, dirt) or with animals are gathering next to each other in a dirt field (error: dirt; infelicities: animals and gathering, which are both pragmatically odd). Clearly, the more the caption changes in the decoy, the easier the task becomes. In contrast, the foil captions we propose only differ from the gold description by one word and are thus more challenging. Secondly, the automatic caption generation of Ding et al means that 'correct' descriptions can be produced, resulting in some confusion in human responses to the task. We made sure to prevent such cases, and human performance on our dataset is thus close to $100 \%$. We note as well that our task does not require any complex instructions for the annotation, indicating that it is intuitive to human beings (see $\S 4$ ). Thirdly, their evaluation is a multiple-choice task, where the system has to compare all captions to understand which one is closest to the image. This is arguably a simpler task than the one we propose, where a caption is given and the system is asked to classify it as correct or foil: as we show in $\S 4$, detecting a correct caption is much easier than detecting foils. So evaluating precision on both gold and foil items is crucial.

Finally, (Johnson et al., 2016) proposed CLEVR, a dataset for the diagnostic evaluation of VQA systems. This dataset was designed with the explicit goal of enabling detailed analysis of different aspects of visual reasoning, by minimising dataset biases and providing rich ground-truth representations for both images and questions.

(iii) Lack of objective evaluation metrics: The evaluation of Natural Language Generation (NLG) systems is known to be a hard problem. It is further unclear whether the quality of LaVi models should be measured using metrics designed for language-only tasks. Elliott and Keller (2014) performed a sentence-level correlation analysis of NLG evaluation measures against expert human judgements in the context of IC. Their study revealed that most of those metrics were only weakly correlated with human judgements. In the same line of research, Anderson et al. (2016) showed that the most widely-used metrics for IC fail to capture semantic propositional content, which is an essential component of human caption evaluation. They proposed a semantic evaluation metric called SPICE, that measures how effectively image captions recover objects, attributes and the relations between them. In this paper, we tackle this problem by proposing tasks which can be evaluated based on objective metrics for classification/detection error.

\section{Dataset}

In this section, we describe how we automatically generate FOIL-COCO datapoints, i.e. image, original and foil caption triples. We used the training and validation Microsoft's Common Objects in Context (MS-COCO) dataset (Lin et al., 2014) (2014 version) as our starting point. In MS-COCO, each image is described by at least five descriptions written by humans via Amazon Mechanical Turk (AMT). The images contains 91 common object categories (e.g. dog, elephant, bird, ... and car, bicycle, airplane, ...), from 11 supercategories (Animal, Vehicle, resp.), with 82 of them having more than $5 \mathrm{~K}$ labeled instances. In total there are 123,287 images with captions (82,783 for training and 40,504 for validation). ${ }^{2}$

Our data generation process consists of four

\footnotetext{
${ }^{2}$ The MS-COCO test set is not available for download.
} 


\begin{tabular}{l|c|c|c|c|} 
& nr. of datapoints & nr. unique images & nr. of tot. captions & nr. target::foil pairs \\
\hline Train & 197,788 & 65,697 & 395,576 & 256 \\
Test & 99,480 & 32,150 & 198,960 & 216
\end{tabular}

Table 1: Composition of FOIL-COCO.

main steps, as described below. The last two steps are illustrated in Figure 2.

1. Generation of replacement word pairs We want to replace one noun in the original caption (the target) with an incorrect but similar word (the foil). To do this, we take the labels of MSCOCO categories, and we pair together words belonging to the same supercategory (e.g., bicycle::motorcycle, bicycle::car, bird::dog). We use as our vocabulary 73 out of the 91 MS-COCO categories, leaving out those categories that are multiword expressions (e.g. traffic light). We thus obtain 472 target::foil pairs.

2. Splitting of replacement pairs into training and testing To avoid the models learning trivial correlations due to replacement frequency, we randomly split, within each supercategory, the candidate target::foil pairs which are used to generate the captions of the training vs. test sets. We obtain 256 pairs, built out of 72 target and 70 foil words, for the training set, and 216 pairs, containing 73 target and 71 foil words, for the test set.

3. Generation of foil captions We would like to generate foil captions by replacing only target words which refer to visually salient objects. To this end, given an image, we replace only those target words that occur in more than one MS-COCO caption associated with that image. Moreover, we want to use foils which are not visually present, i.e. that refer to visual content not present in the image. Hence, given an image, we only replace a word with foils that are not among the labels (objects) annotated in MS-COCO for that image. We use the images from the MS-COCO training and validation sets to generate our training and test sets, respectively. We obtain 2,229,899 for training and 1,097,012 captions for testing.

4. Mining the hardest foil caption for each image To eliminate possible visual-language dataset bias, out of all foil captions generated in step 3, we select only the hardest one. For this purpose, we need to model the visual-language bias of the dataset. To this end, we use Neuraltalk ${ }^{3}$

\footnotetext{
${ }^{3}$ https://github.com/karpathy/ neuraltalk
}

(Karpathy and Fei-Fei, 2015), one of the stateof-the-art image captioning systems, pre-trained on MS-COCO. Neuraltalk is based on an LSTM which takes as input an image and generates a sentence describing its content. We obtain a neural network $\mathcal{N}$ that implicitly represents the visuallanguage bias through its weights. We use $\mathcal{N}$ to approximate the conditional probability of a caption $C$ given a dataset $T$ and and an image $I$ $(P(C \mid I, T))$. This is obtained by simply using the loss $l(C, \mathcal{N}(I))$ i.e., the error obtained by comparing the pseudo-ground truth $C$ with the sentence predicted by $\mathcal{N}: P(C \mid I, T)=1-l(C, \mathcal{N}(I))$ (we refer to (Karpathy and Fei-Fei, 2015) for more details on how $l()$ is computed). $P(C \mid I, T)$ is used to select the hardest foil among all the possible foil captions, i.e. the one with the highest probability according to the dataset bias learned by $\mathcal{N}$. Through this process, we obtain 197,788 and 99,480 original::foil caption pairs for the training and test sets, respectively. None of the target::foil word pairs are filtered out by this mining process.

The final FOIL-COCO dataset consists of 297,268 datapoints (197,788 in training and 99,480 in test set). All the 11 MS-COCO supercategories are represented in our dataset and contain 73 categories from the 91 MS-COCO ones (4.8 categories per supercategory on average.) Further details are reported in Table 1.

\section{Experiments and Results}

We conduct three tasks, as presented below:

Task 1 (T1): Correct vs. foil classification Given an image and a caption, the model is asked to mark whether the caption is correct or wrong. The aim is to understand whether LaVi models can spot mismatches between their coarse representations of language and visual input.

Task 2 (T2): Foil word detection Given an image and a foil caption, the model has to detect the foil word. The aim is to evaluate the understanding of the system at the word level. In order to systematically check the system's performance with different prior information, we test two different set- 


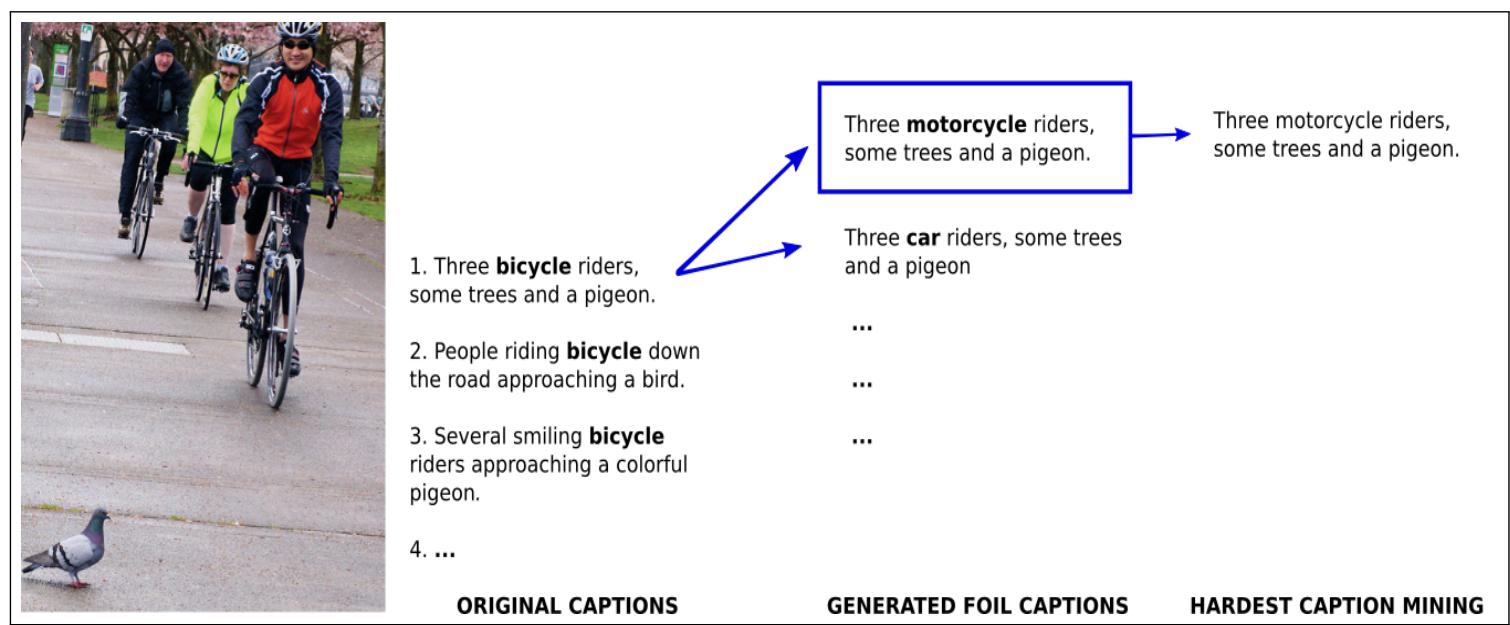

Figure 2: The main aspects of the foil caption generation process. Left column: some of the original COCO captions associated with an image. In bold we highlight one of the target words (bicycle), chosen because it is mentioned by more than one annotator. Middle column: For each original caption and each chosen target word, different foil captions are generated by replacing the target word with all possible candidate foil replacements. Right column: A single caption is selected amongst all foil candidates. We select the 'hardest' caption, according to Neuraltalk model, trained using only the original captions.

tings: the foil has to be selected amongst (a) only the nouns or (b) all content words in the caption.

Task 3 (T3): Foil word correction Given an image, a foil caption and the foil word, the model has to detect the foil and provide its correction. The aim is to check whether the system's visual representation is fine-grained enough to be able to extract the information necessary to correct the error. For efficiency reasons, we operationalise this task by asking models to select a correction from the set of target words, rather than the whole dataset vocabulary (viz. more than $10 \mathrm{~K}$ words).

\subsection{Models}

We evaluate both VQA and IC models against our tasks. For the former, we use two of the three models evaluated in (Goyal et al., 2016a) against a balanced VQA dataset. For the latter, we use the multimodal bi-directional LSTM, proposed in (Wang et al., 2016), and adapted for our tasks.

LSTM + norm I: We use the best performing VQA model in (Antol et al., 2015) (deeper LSTM + norm I). This model uses a two stack LongShort Term Memory (LSTM) to encode the questions and the last fully connected layer of VGGNet to encode images. Both image embedding and caption embedding are projected into a 1024dimensional feature space. Following (Antol et al., 2015), we have normalised the image feature before projecting it. The combination of these two projected embeddings is performed by a pointwise multiplication. The multi-model representation thus obtained is used for the classification, which is performed by a multi-layer perceptron (MLP) classifier.

HieCoAtt: We use the Hierarchical CoAttention model proposed by ( $\mathrm{Lu}$ et al., 2016) that co-attends to both the image and the question to solve the task. In particular, we evaluate the 'alternate' version, i.e. the model that sequentially alternates between generating some attention over the image and question. It does so in a hierarchical way by starting from the word-level, then going to the phrase and then to the entire sentence-level. These levels are combined recursively to produce the distribution over the foil vs. correct captions.

IC-Wang: Amongst the IC models, we choose the multimodal bi-directional LSTM (Bi-LSTM) model proposed in (Wang et al., 2016). This model predicts a word in a sentence by considering both the past and future context, as sentences are fed to the LSTM in forward and backward order. The model consists of three modules: a CNN for encoding image inputs, a Text-LSTM (T-LSTM) for encoding sentence inputs, a Multimodal LSTM (M-LSTM) for embedding visual and textual vectors to a common semantic space and decoding to sentence. The bidirectional LSTM is implemented with two separate LSTM layers. 
Baselines: We compare the SoA models above against two baselines. For the classification task, we use a Blind LSTM model followed by a fully connected layer and softmax and train it only on captions as input to predict the answer. In addition, we evaluate the CNN+LSTM model, where visual and textual features are simply concatenated.

The models at work on our three tasks For the classification task (T1), the baselines and VQA models can be applied directly. We adapt the generative IC model to perform the classification task as follows. Given a test image $I$ and a test caption, for each word $w_{t}$ in the test caption, we remove the word and use the model to generate new captions in which the $w_{t}$ has been replaced by the word $v_{t}$ predicted by the model $\left(w_{1}, \ldots, w_{t-1}, v_{t}, w_{t-1}, \ldots, w_{n}\right)$. We then compare the conditional probability of the test caption with all the captions generated from it by replacing $w_{t}$ with $v_{t}$. When all the conditional probabilities of the generated captions are lower than the one assigned to the test caption the latter is classified as good, otherwise as foil. For the other tasks, the models have been trained on T1. To perform the foil word detection task (T2), for the VQA models, we apply the occlusion method. Following (Goyal et al., 2016b), we systematically occlude subsets of the language input, forward propagate the masked input through the model, and compute the change in the probability of the answer predicted with the unmasked original input. For the IC model, similarly to T1, we sequentially generate new captions from the foil one by replacing, one by one, the words in it and computing the conditional probability of the foil caption and the one generated from it. The word whose replacement generate the caption with the highest conditional probabilities is taken to be the foil word. Finally, to evaluate the models on the error correction task (T3), we apply the linear regression method over all the target words and select the target word which has the highest probability of making that wrong caption correct with respect to the given image.

Upper-bound Using Crowdflower, we collected human answers from 738 native English speakers for 984 image-caption pairs randomly selected from the test set. Subjects were given an image and a caption and had to decide whether it was correct or wrong (T1). If they thought it was wrong, they were required to mark the error in the caption (T2). We collected 2952 judgements (i.e. 3 judgements per pair and 4 judgements per rater) and computed human accuracy in $\mathrm{T} 1$ when considering as answer (a) the one provided by at least 2 out of 3 annotators (majority) and (b) the one provided by all 3 annotators (unanimity). The same procedure was adopted for computing accuracies in $\mathrm{T} 2$. Accuracies in both $\mathrm{T} 1$ an $\mathrm{T} 2$ are reported in Table 2. As can be seen, in the $m a$ jority setting annotators are quasi-perfect in classifying captions $(92.89 \%)$ and detecting foil words (97.00\%). Though lower, accuracies in the unanimity setting are still very high, with raters providing the correct answer in 3 out of 4 cases in both tasks. Hence, although we have collected human answers only on a rather small subset of the test set, we believe their results are representative of how easy the tasks are for humans.

\subsection{Results}

As shown in Table 2, the FOIL-COCO dataset is challenging. On $\mathrm{T} 1$, for which the chance level is $50.00 \%$, the 'blind', language-only model, does badly with an accuracy of $55.62 \%(25.04 \%$ on foil captions), demonstrating that language bias is minimal. By adding visual information, CNN+LSTM, the overall accuracy increases by $5.45 \%$ (7.94\% on foil captions.) reaching $61.07 \%$ (resp. 32.98\%). Both SoA VQA and IC models do significantly worse than humans on both $\mathrm{T} 1$ and T2. The VQA systems show a strong bias towards correct captions and poor overall performance. They only identify $34.51 \%$ (LSTM +norm I) and $36.38 \%$ (HieCoAtt) of the incorrect captions (T1). On the other hand, the IC model tends to be biased toward the foil captions, on which it achieves an accuracy of $45.44 \%$, higher than the VQA models. But the overall accuracy (42.21\%) is poorer than the one obtained by the two baselines. On the foil word detection task, when considering only nouns as possible foil word, both the IC and the LSTM+norm I models perform close to chance level, and the HieCoAtt performs somewhat better, reaching $38.79 \%$. Similar results are obtained when considering all words in the caption as possible foil. Finally, the VQA models' accuracy on foil word correction (T3) is extremely low, at $4.7 \%$ (LSTM + norm I) and $4.21 \%$ (HieCoAtt). The result on T3 makes it clear that the VQA systems are unable to extract from the image rep- 
resentation the information needed to correct the foil: despite being told which element in the caption is wrong, they are not able to zoom into the correct part of the image to provide a correction, or if they are, cannot name the object in that region. The IC model performs better compared to the other models, having an accuracy that is $20,78 \%$ higher than chance level.

\begin{tabular}{|l|c|c|c|}
\hline \multicolumn{4}{|c|}{ T1: Classification task } \\
\hline & Overall & Correct & Foil \\
\hline Blind & 55.62 & 86.20 & 25.04 \\
CNN+LSTM & 61.07 & 89.16 & 32.98 \\
\hline IC-Wang & 42.21 & 38.98 & 45.44 \\
\hline LSTM + norm I & 63.26 & $\mathbf{9 2 . 0 2}$ & 34.51 \\
HieCoAtt & $\mathbf{6 4 . 1 4}$ & 91.89 & $\mathbf{3 6 . 3 8}$ \\
\hline Human (majority) & 92.89 & 91.24 & 94.52 \\
Human (unanimity) & 76.32 & 73.73 & 78.90 \\
\hline
\end{tabular}

\begin{tabular}{|l|c|c|}
\hline \multicolumn{3}{|c|}{ T2: Foil word detection task } \\
\hline & nouns & all content words \\
\hline Chance & 23.25 & 15.87 \\
\hline IC-Wang & 27.59 & 23.32 \\
\hline LSTM + norm I & 26.32 & 24.25 \\
HieCoAtt & $\mathbf{3 8 . 7 9}$ & $\mathbf{3 3 . 6 9}$ \\
\hline Human (majority) & & 97.00 \\
Human (unanimity) & & 73.60 \\
\hline
\end{tabular}

\begin{tabular}{|l|c|}
\hline \multicolumn{2}{|c|}{ T3: Foil word correction task } \\
\hline & all target words \\
\hline Chance & 1.38 \\
\hline IC-Wang & $\mathbf{2 2 . 1 6}$ \\
\hline LSTM + norm I & 4.7 \\
HieCoAtt & 4.21 \\
\hline
\end{tabular}

Table 2: T1: Accuracy for the classification task, relatively to all image-caption pairs (overall) and by type of caption (correct vs. foil); T2: Accuracy for the foil word detection task, when the foil is known to be among the nouns only or when it is known to be among all the content words; T3: Accuracy for the foil word correction task when the correct word has to be chosen among any of the target words.

\section{Analysis}

We performed a mixed-effect logistic regression analysis in order to check whether the behavior of the best performing models in T1, namely the VQA models, can be predicted by various linguis- tic variables. We included: 1) semantic similarity between the original word and the foil (computed as the cosine between the two corresponding word2vec embeddings (Mikolov et al., 2013)); 2) frequency of original word in FOIL-COCO captions; 3) frequency of the foil word in FOILCOCO captions; 4) length of the caption (number of words). The mixed-effect model was performed to get rid of possible effects due to either object supercategory (indoor, food, vehicle, etc.) or target::foil pair (e.g., zebra::giraffe, boat::airplane, etc.). For both LSTM + norm I and HieCoAtt, word2 vec similarity, frequency of the original word, and frequency of the foil word turned out to be highly reliable predictors of the model's response. The higher the values of these variables, the more the models tend to provide the wrong output. That is, when the foil word (e.g. cat) is semantically very similar to the original one (e.g. $d o g$ ), the models tend to wrongly classify the caption as 'correct'. The same holds for frequency values. In particular, the higher the frequency of both the original word and the foil one, the more the models fail. This indicates that systems find it difficult to distinguish related concepts at the textvision interface, and also that they may tend to be biased towards frequently occurring concepts, 'seeing them everywhere' even when they are not present in the image. Caption length turned out to be only a partially reliable predictor in the LSTM + norm I model, whereas it is a reliable predictor in HieCoAtt. In particular, the longer the caption, the harder for the model to spot that there is a foil word that makes the caption wrong.

As revealed by the fairly high variance explained by the random effect related to target::foil pairs in the regression analysis, both models perform very well on some target::foil pairs, but fail on some others (see leftmost part of Table 4 for same examples of easy/hard target::foil pairs). Moreover, the variance explained by the random effect related to object supercategory is reported in Table 3. As can be seen, for some supercategories accuracies are significatively higher than for others (compare, e.g., 'electronic' and 'outdoor').

In a separate analysis, we also checked whether there was any correlation between results and the position of the foil in the sentence, to ensure the models did not profit from any undesirable artifacts of the data. We did not find any such correlation. 


\begin{tabular}{|c|c|c|c|c|}
\hline Super-category & No. of object & $\begin{array}{c}\text { No. of foil } \\
\text { captions }\end{array}$ & $\begin{array}{c}\text { Acc. using } \\
\text { LSTM + norm I }\end{array}$ & $\begin{array}{c}\text { Acc. using } \\
\text { HieCoAtt }\end{array}$ \\
\hline outdoor & 2 & 107 & 2.80 & 0.93 \\
\hline food & 9 & 10407 & 22.00 & 26.59 \\
\hline indoor & 6 & 4911 & 30.74 & 27.97 \\
\hline appliance & 5 & 2811 & 32.72 & 34.54 \\
\hline sports & 10 & 16276 & 31.57 & 31.61 \\
\hline animal & 10 & 21982 & 39.03 & 43.18 \\
\hline vehicle & 8 & 16514 & 34.38 & 40.09 \\
\hline furniture & 5 & 13625 & 33.27 & 33.13 \\
\hline accessory & 5 & 3040 & 49.53 & 31.80 \\
\hline electronic & 6 & 5615 & 45.82 & 43.47 \\
\hline kitchen & 7 & 4192 & 38.19 & 45.34 \\
\hline
\end{tabular}

Table 3: Classification Accuracy of foil captions by Super Categories (T1). The No. of the objects and the No. of foil captions refer to the test set. The training set has a similar distribution.

\begin{tabular}{|c|c|c|c|c|c|c|c|}
\hline \multicolumn{2}{|l|}{ Top-5 } & \multicolumn{2}{|l|}{ Bottom-5 } & \multicolumn{2}{|l|}{ Top-5 } & \multicolumn{2}{|l|}{ Bottom-5 } \\
\hline \multicolumn{4}{|c|}{ T1: LSTM + norm I } & \multicolumn{4}{|c|}{ T2: LSTM + norm I } \\
\hline racket::glove & 100 & motorcycle::airplane & 0 & drier::scissors & 100 & glove::skis & 0 \\
\hline racket::kite & 97.29 & bicycle::airplane & 0 & zebra::giraffe & 88.98 & snowboard::racket & 0 \\
\hline couch::toilet & 97.11 & drier::scissors & 0 & boat::airplane & 87.87 & donut::apple & 0 \\
\hline racket::skis & 95.23 & bus::airplane & 0.35 & truck::airplane & 85.71 & glove::surfboard & 0 \\
\hline giraffe::sheep & 95.09 & zebra::giraffe & 0.43 & train::airplane & 81.93 & spoon::bottle & 0 \\
\hline \multicolumn{4}{|c|}{ T1: HieCoAtt } & \multicolumn{4}{|c|}{ T2: HieCoAtt } \\
\hline tie::handbag & 100 & drier::scissors & 0 & zebra::elephant & 94.92 & drier::scissors & 0 \\
\hline snowboard::glove & 100 & fork::glass & 0 & backpack::handbag & 94.44 & handbag::tie & 0 \\
\hline racket::skis & 100 & handbag::tie & 0 & cow::zebra & 93.33 & broccoli:orange & 1.47 \\
\hline racket::glove & 100 & motorcycle::airplane & 0 & bird::sheep & 93.11 & zebra::giraffe & 1.96 \\
\hline backpack::handbag & 100 & train::airplane & 0 & orange::carrot & 92.37 & boat::airplane & 2.09 \\
\hline
\end{tabular}

Table 4: Easiest and hardest target::foil pairs: T1 (caption classification) and T2 (foil word detection).

To better understand results on $\mathrm{T} 2$, we performed an analysis investigating the performance of the VQA models on different target::foil pairs. As reported in Table 4 (right), both models perform nearly perfectly with some pairs and very badly with others. At first glance, it can be noticed that LSTM + norm I is very effective with pairs involving vehicles (airplane, truck, etc.), whereas HieCoAtt seems more effective with pairs involving animate nouns (i.e. animals), though more in depth analysis is needed on this point. More interestingly, some pairs that are found to be predicted almost perfectly by LSTM + I norm, namely boat::airplane, zebra::giraffe, and drier::scissors, turn out to be among the Bottom-5 cases in HieCoAtt. This suggests, on the one hand, that the two VQA models use different strategies to perform the task. On the other hand, it shows that our dataset does not contain cases that are a priori easy for any model.

The results of IC-Wang on T3 are much higher than LSTM + norm I and HieCoAtt, although it is outperformed by or is on par with HieCoAtton on T1-T2. Our interpretation is that this behaviour is related to the discriminative/generative nature of our tasks. Specifically, T1 and T2 are discriminative tasks and LSTM + norm I and HieCoAtt are discriminative models. Conversely, T3 is a generative task (a word needs to be generated) and IC-Wang is a generative model. It would be interesting to test other IC models on T3 and compare their results against the ones reported here. However, note that IC-Wang is 'tailored' for T3 because it takes as input the whole sentence (minus the word to be generated), while common sequential IC approaches can only generate a word depending on the previous words in the sentence.

As far as human performance is concerned, both $\mathrm{T} 1$ and $\mathrm{T} 2$ turn out to be extremely easy. In T1, image-caption pairs were correctly judged as correct/wrong in overall 914 out of 984 cases (92.89\%) in the majority setting. In the unanim- 
ity setting, the correct response was provided in 751 out of 984 cases (76.32\%). Judging foil captions turns out to be slightly easier than judging correct captions in both settings, probably due to the presence of typos and misspellings that sometimes occur in the original caption (e.g. raters judge as wrong the original caption People playing ball with a drown and white dog, where 'brown' was misspelled as 'drown'). To better understand which factors contribute to make the task harder, we qualitatively analyse those cases where all annotators provided a wrong judgement for an image-caption pair. As partly expected, almost all cases where original captions (thus correct for the given image) are judged as being wrong are cases where the original caption is indeed incorrect. For example, a caption using the word 'motorcycle' to refer to a bicycle in the image is judged as wrong. More interesting are those cases where all raters agreed in considering as correct image-caption pairs that are instead foil. Here, it seems that vagueness as well as certain metaphorical properties of language are at play: human annotators judged as correct a caption describing Blue and banana large birds on tree with metal pot (see Fig 3, left), where 'banana' replaced 'orange'. Similarly, all raters judged as correct the caption $A$ cat laying on a bed next to an opened keyboard (see Fig 3, right), where the cat is instead laying next to an opened laptop.

Focusing on $\mathrm{T} 2$, it is interesting to report that among the correctly-classified foil cases, annotators provided the target word in $97 \%$ and $73.6 \%$ of cases in the majority and unanimity setting, respectively. This further indicates that finding the foil word in the caption is a rather trivial task for humans.

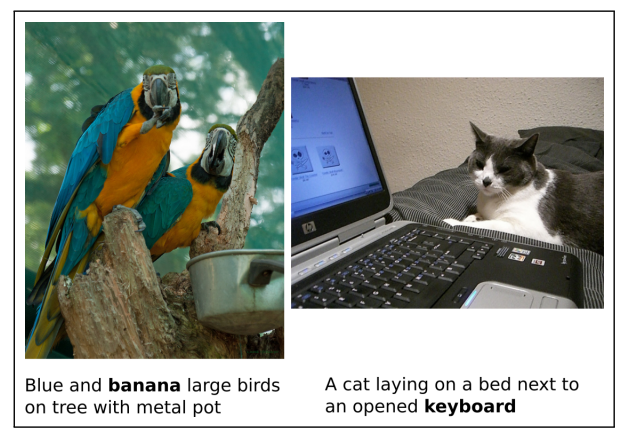

Figure 3: Two cases of foil image-caption pairs that are judged as correct by all annotators.

\section{Conclusion}

We have introduced FOIL-COCO, a large dataset of images associated with both correct and foil captions. The error production is automatically generated, but carefully thought out, making the task of spotting foils particularly challenging. By associating the dataset with a series of tasks, we allow for diagnosing various failures of current $\mathrm{LaVi}$ systems, from their coarse understanding of the correspondence between text and vision to their grasp of language and image structure.

Our hypothesis is that systems which, like humans, deeply integrate the language and vision modalities, should spot foil captions quite easily. The SoA LaVi models we have tested fall through that test, implying that they fail to integrate the two modalities. To complete the analysis of these results, we plan to carry out a further task, namely ask the system to detect in the image the area that produces the mismatch with the foil word (the red box around the bird in Figure 1.) This extra step would allow us to fully diagnose the failure of the tested systems and confirm what is implicit in our results from task 3: that the algorithms are unable to map particular elements of the text to their visual counterparts. We note that the addition of this extra step will move this work closer to the textual/visual explanation research (e.g., (Park et al., 2016; Selvaraju et al., 2016)). We will then have a pipeline able to not only test whether a mistake can be detected, but also whether the system can explain its decision: 'the wrong word is $d o g$ because the cyclists are in fact approaching a bird, there, in the image'.

LaVi models are a great success of recent research, and we are impressed by the amount of ideas, data and models produced in this stimulating area. With our work, we would like to push the community to think of ways that models can better merge language and vision modalites, instead of merely using one to supplement the other.

\section{Acknowledgments}

We are greatful to the Erasmus Mundus European Master in Language and Communication Technologies (EM LCT) for the scholarship provided to the third author. Moreover, we gratefully acknowledge the support of NVIDIA Corporation with the donation of the GPUs used in our research. 


\section{References}

Aishwarya Agrawal, Dhruv Batra, and Devi Parikh. 2016. Analyzing the behavior of visual question answering models. In Proceedings of Empirical Methods in Natural Language Processing (EMNLP).

Peter Anderson, Basura Fernando, Mark Johnson, and Stephen Gould. 2016. SPICE: Semantic Propositional Image Caption Evaluation. In In Proceedings of the European Conference on Computer Vi$\operatorname{sion}(E C C V)$.

Stanislaw Antol, Aishwarya Agrawal, Jiasen Lu, Margaret Mitchell, Dhruv Batra, C. Lawrence Zitnick, and Devi Parikh. 2015. VQA: Visual Question Answering. In International Conference on Computer Vision (ICCV). https://github.com/ VT-vision-lab/VQA_LSTM_CNN.

Raffaella Bernardi, Ruket Cakici, Desmond Elliott, Aykut Erdem, Erkut Erdem, Nazli Ikizler-Cinbis, Frank Keller, Adrian Muscat, and Barbara Plank. 2016. Automatic description generation from images: A survey of models, datasets, and evaluation measures. J. Artif. Intell. Res.(JAIR) 55:409-442.

Xinlei Chen and C Lawrence Zitnick. 2015. Mind's eye: A recurrent visual representation for image caption generation. In Proceedings of the IEEE Conference on Computer Vision and Pattern Recognition. pages 2422-2431.

Nan Ding, Sebastian Goodman, Fei Sha, and Radu Soricut. 2016. Understanding image and text simultaneously: a dual vision-language machine comprehension task. arXiv preprint arXiv:1612.07833 .

Jeffrey Donahue, Lisa Anne Hendricks, Sergio Guadarrama, Marcus Rohrbach, Subhashini Venugopalan, Kate Saenko, and Trevor Darrell. 2015. Long-term recurrent convolutional networks for visual recognition and description. In Proceedings of the IEEE conference on computer vision and pattern recognition. pages 2625-2634.

Desmond Elliott and Frank Keller. 2014. Comparing automatic evaluation measures for image description. In In Proceedings of the 52nd Annual Meeting of the Association for Computational Linguistics: Short Papers. pages 452-457.

Hao Fang, Saurabh Gupta, Forrest Iandola, Rupesh K Srivastava, Li Deng, Piotr Dollár, Jianfeng Gao, Xiaodong He, Margaret Mitchell, John C Platt, et al. 2015. From captions to visual concepts and back. In Proceedings of the IEEE Conference on Computer Vision and Pattern Recognition. pages 1473-1482.

Haoyuan Gao, Junhua Mao, Jie Zhou, Zhiheng Huang, Lei Wang, and Wei Xu. 2015. Are you talking to a machine? dataset and methods for multilingual image question. In Advances in Neural Information Processing Systems. pages 2296-2304.
Yash Goyal, Tejas Khot, Douglas Summers-Stay, Dhruv Batra, and Devi Parikh. 2016a. Making the $\mathrm{V}$ in VQA Matter: Elevating the Role of Image Understanding in Visual Question Answering. arXiv preprint arXiv: 1612.00837 .

Yash Goyal, Akrit Mohapatra, Devi Parikh, and Dhruv Batra. 2016b. Towards Transparent AI Systems: Interpreting Visual Question Answering Models . In In Proceedings of ICML Visualization Workshop.

Micah Hodosh and Julia Hockenmaier. 2016. Focused evaluation for image description with binary forcedchoice tasks. In Proceedings of the 5th Workshop on Vision and Language (VL'16).

Micah Hodosh, Peter Young, and Julia Hockenmaier. 2013. Framing image description as a ranking task: Data, models and evaluation metrics. Journal of Artificial Intelligence Research 47:853-899.

Allan Jabri, Armand Joulin, and Laurens van der Maaten. 2016. Revisiting visual question answering baselines. In Proceedings of the European Conference on Computer Vision (ECCV). pages 727-739.

Justin Johnson, Bharath Hariharan, Laurens van der Maaten, Li Fei-Fei, C. Lawrence Zitnick, and Ross Girshick. 2016. CLEVR: A Diagnostic Dataset for Compositional Language and Elementary Visual Reasoning. ArXiv:1612.06890.

Kushal Kafle and Christopher Kanan. 2016. Visual question answering: Datasets, algorithms, and future challenges. arXiv preprint arXiv:1610.01465.

Andrej Karpathy and Li Fei-Fei. 2015. Deep visualsemantic alignments for generating image descriptions. In Proceedings of the IEEE Conference on Computer Vision and Pattern Recognition. pages 3128-3137.

Tsung-Yi Lin, Michael Maire, Serge Belongie, James Hays, Pietro Perona, Deva Ramanan, Piotr Dollár, and C Lawrence Zitnick. 2014. Microsoft COCO: Common Objects in Context. In European Conference on Computer Vision. Springer, pages 740-755.

Jiasen Lu, Jianwei Yang, Dhruv Batra, and Devi Parikh. 2016. Hierarchical question-image coattention for visual question answering. In In Proceedings of NIPS 2016. https://github. com/jiasenlu/HieCoAttenVQA.

Mateusz Malinowski and Mario Fritz. 2014. A multiworld approach to question answering about realworld scenes based on uncertain input. In Advances in Neural Information Processing Systems. pages 1682-1690.

Mateusz Malinowski, Marcus Rohrbach, and Mario Fritz. 2015. Ask your neurons: A neural-based approach to answering questions about images. In Proceedings of the IEEE International Conference on Computer Vision. pages 1-9. 
Tomas Mikolov, Kai Chen, Greg Corrado, and Jeffrey Dean. 2013. Efficient estimation of word representations in vector space. arXiv preprint arXiv:1301.3781 .

Dong Huk Park, Lisa Anne Hendricks, Zeynep Akata, Trevor Darrell Bernt Schiele, and Marcus Rohrbach. 2016. Attentive explanations: Justifying decisions and pointing to the evidence. ArXiv:1612.04757.

Mengye Ren, Ryan Kiros, and Richard Zemel. 2015. Exploring models and data for image question answering. In Advances in Neural Information Processing Systems (NIPS 2015).

Ramprasaath R. Selvaraju, Abhishek Das, Ramakrishna Vedantam, Michael Cogswell, Devi Parikh, and Dhruv Batra. 2016. Grad-cam: Why did you say that? visual explanations from deep networks via gradient-based localization. ArXiv:1610.02391v2.

Oriol Vinyals, Alexander Toshev, Samy Bengio, and Dumitru Erhan. 2015. Show and tell: A neural image caption generator. In Proceedings of the IEEE Conference on Computer Vision and Pattern Recognition. pages 3156-3164.

Cheng Wang, Haojin Yang, Christian Bartz, and Christoph Meinel. 2016. Image captioning with deep bidirectional LSTMs. In Proceedings of the 2016 ACM on Multimedia Conference. ACM, pages 988-997.

Qi Wu, Damien Teney, Peng Wang, Chunhua Shen, Anthony Dick, and Anton van den Hengel. 2016. Visual question answering: A survey of methods and datasets. arXiv preprint arXiv:1607.05910 .

Peter Young, Alice Lai, Micah Hodosh, and Julia Hockenmaier. 2014. From image descriptions to visual denotations: New similarity metrics for semantic inference over event descriptions. Transactions of the Association for Computational Linguistics 2:67-78.

Peng Zhang, Yash Goyal, Douglas Summers-Stay, Dhruv Batra, and Devi Parikh. 2016. Yin and yang: Balancing and answering binary visual questions. In Proceedings of the IEEE Conference on Computer Vision and Pattern Recognition. pages 5014-5022.

Bolei Zhou, Yuandong Tian, Sainbayar Sukhbaatar, Arthur Szlam, and Rob Fergus. 2015. Simple baseline for visual question answering. arXiv preprint arXiv:1512.02167. 